\title{
Guide antérieur, \\ impératifs physiologiques \\ et restauration prothétique
}

\section{RÉSUMÉ}

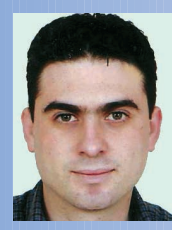

Omar IRAQUI

Spécialiste en prothèse adjointe, Appt 7, Imm. 39, rue Oued Tensift,

Agdal, Rabat, Maroc.

Salwa BERRADA

Professeur d'Enseignement Supérieur, Service de prothèse adjointe,

Université M ohamed V,

Faculté de médecine dentaire de Rabat,

BP 6212, Rabat-Instituts, Maroc.

\section{Nadia MERZOUK}

Professeur d'Enseignement supérieur, Service de prothèse adjointe,

Université M ohamed V,

Faculté de médecine dentaire de Rabat, BP 6212, Rabat-Instituts, Maroc.

\section{Ahmed ABDEDINE}

Professeur d'Enseignement Supérieure, Chef du service de prothèse adjointe, Université Mohamed V,

Faculté de médecine dentaire de Rabat, BP 6212, Rabat-Instituts, Maroc.

L'équilibre du système masticateur est une priorité aux yeux de tout praticien occluso-conscient, et nécessite la restauration d'un guidage antérieur fonctionnel et protecteur. Cet objectif ne peut être atteint que par l'examen et le diagnostic des rapports occlusaux antérieurs par comparaison à un modèle académique de référence. L'attitude thérapeutique adoptée sera modulée en fonction des données recueillies et fait appel à l'intégration, la recopie ou la recréation du guidage. Quelle que soit la séquence thérapeutique, la validation des paramètres de guidage par des prothèses provisoires, durant une phase de temporisation et la retranscription de ces données sur les prothèses définitives sont les seuls garants de l'intégration prothétique.

Ce travail se propose, de mettre en évidence l'intérêt d'un guide antérieur fonctionnel et les moyens d'y parvenir dans le cadre d'une restauration prothétique par prothèse fixée.

- guide antérieur

- occlusion

- prothèse 


\section{Introduction}

$>$

La santé de I'appareil manducateur

dépend de l'équilibre et de I'harmonie fonctionnelle de ses différents constituants dentaires, parodontaux, neuro-musculaires et articulaires. Le système neuro-musculaire, véritable chef d'orchestre de cette harmonie[1], exerce son contrôle grâce aux afférences sensi- tives provenant, entre autre, de propriocepteurs desmodontaux[2].

La sensibilité proprioceptive atteint son maximum au niveau des dents antérieures, leur conférant un rôle privilégié dans l'équilibre du système masticateur[2,3]. Le guide antérieur prend alors toute son importance.

\section{Guide antérieur et rôle de protection}

Le guide antérieur (G.A.) est généralement défini comme "l'influence» des contacts antérieurs sur les mouvements mandibulaires. $\mathrm{Ce}$ concept décrit la prise en charge des mouvements mandibulaires de et vers I'OIM, jusqu'aux positions de bout à bout incisif en propulsion, et canine en latéralité. Slavicek a proposé d'y intégrer le guide anti-rétropulsif, représentant la prise en charge du mouvement mandibulaire entre OIM et ORC lorsque les deux positions sont distinctes[4].

Au-delà des différentes fonctions qui lui sont dévolues[5], le guide antérieur exerce une action protectrice de par ses composantes biomécaniques et proprioceptives qui ne seront individualisées qu'à but didactique[6, 7].

\section{> La protection biomécanique}

Les dents postérieures assument les charges fonctionnelles en PIM, les dents antérieures prennent en charges les excursions mandibulaires assurant une désocclusion postérieure. Cette configuration transforme le système masticateur en un levier de $3^{e}$ genre (fig. 1), ce qui permet le fonctionnement du système avec un minimum d'usure: plus les contacts dentaires sont situés antérieurement sur l'arcade, plus faible est la charge transmise aux éléments biologiques, dentaires ou parodontaux $[3,6]$. Inversement, plus les contacts sont postérieurs, plus les contraintes dentaires (abrasions), parodontales (trauma occlusal), musculaires (hyperactivité) et articulaires (contraintes) sont importantes. Cette notion est confirmée par Dawson[8] qui soutient que même si le parodonte du secteur antérieur est affaibli, il reste préférable de lui faire assurer la fonction de désocclusion postérieure. Celle-ci

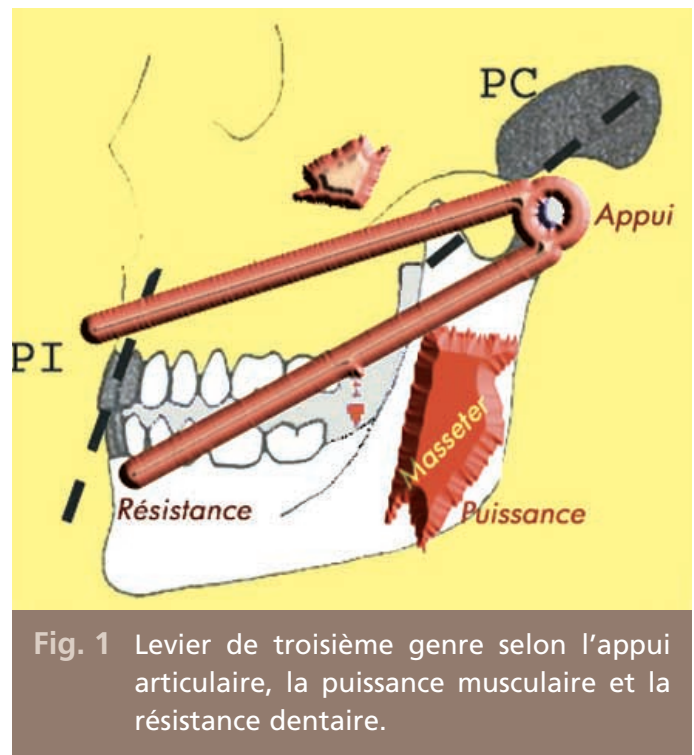


réduirait la contraction musculaire soulageant par là même les dents antérieures. Les travaux de Manns et coll.[9] montrent que la désocclusion des dents cuspidées implique une diminution de pression au niveau des dents antérieures, de plus il y aurait une moindre activité des muscles élévateurs.

Le désengrènement des dents postérieures, provoqué en partie par les pentes antérieures fonctionnelles ainsi que la pente condylienne (fig. 1), ne doit pas être excessif sous peine d'hyperactivité musculaire et articulaire[3]. En effet, une désocclusion minimale permet une efficacité masticatoire optimale[6, 10].

\section{> La protection proprioceptive}

Les conditions anatomiques des dents antérieures (gracilité des racines, orientations défavorables, fragilité des tissus osseux de soutien) font que les dents antéro-maxillaires ne pourraient pas résister aux contraintes occlusales uniquement grâce à leur avantage mécanique «À la vision mécaniste du guidage dentaire, doit s'ajouter la composante proprioceptive» Orthlieb[4].

La qualité proprioceptive desmodontale décroissante de l'incisive centrale à la dernière molaire, associée à un seuil de détection plus bas pour les forces à direction horizontale, transforment les dents antérieures en véritables palpeurs extéroceptifs. Ainsi, à la manière de la canne blanche de l'aveugle[11], ces contacts permettent au système nerveux central de programmer ou reprogrammer I'amplitude des mouvements mandibulaires et la vitesse d'impact selon des trajectoires libres de tout obstacle préservant les structures dentaires, parodontales, musculaires et articulaires contre les contraintes excessives[4]. Cette liberté assure un fonctionnement réflexe et une facilitation neuro-musculaire par systématisation des fonctions. Lubespère[6] explique que le guidage des déplacements mandibulaires doit être préférentiellement antérieur, du fait de la plus grande sensibilité proprioceptive de cette zone et son importance dans la programmation de l'enveloppe de fonction, et de l'éloignement des effecteurs musculaires (levier de troisième genre)[11].

\section{Evaluation

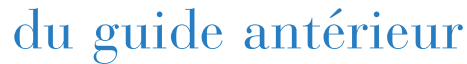

Il est nécessaire d'apprécier la valeur fonctionnelle du guidage antérieur existant par comparaison à un modèle académique idéal de référence[11]. Les éléments d'architecture occlusale du guide antérieur idéal décrits cidessous, sont à la fois critères de diagnostic et règles de construction occlusale.

\section{> Occlusion statique et contacts antérieurs}

La restauration d'un calage postérieur stable est un élément déterminant dans l'établissement d'une occlusion antérieure physiologique et durable. En effet, la perte des dents postérieures provoque des surcharges occlu-

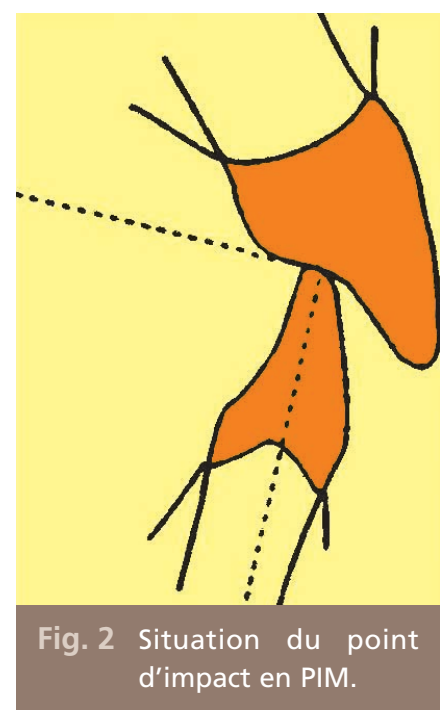


sales antérieures et compromet les structures dentaires, parodontales et neuro-musculo-articulaires du patient[9].

Les contacts antérieurs en OIM peuvent ne pas exister sur toutes les dents, et doivent être légers et symétriques par rapport au plan sagittal médian[10]. Lorsqu'ils existent, ils participent à la stabilisation sagittale et transversale de la mandibule en OIM[7] et permettent la prise en charge immédiate des excursions mandibulaires. On estime qu'il n'existe pas de contacts en OIM passive (inocclusion de $0,1 \mathrm{~mm}$ ) mais des contacts en occlusion forcée active, situés idéalement au point d'inflexion de la courbure linguale (fig. 2) pour une axialisation des forces[4, 9]. L'intercuspidation doit se faire sans la moindre mobilisation des dents antérieures. La recherche de "frémitus» permet d'identifier une altération fonctionnelle du guide antérieur.

\section{Prise en charge des excursions mandibulaires}

\section{$>$ En propulsion}

Le guide incisif oriente le déplacement mandibulaire de I'OIM au bout à bout incisif. Les surfaces palatines antérieures décrivent un entonnoir ouvert vers l'avant, symétrique par

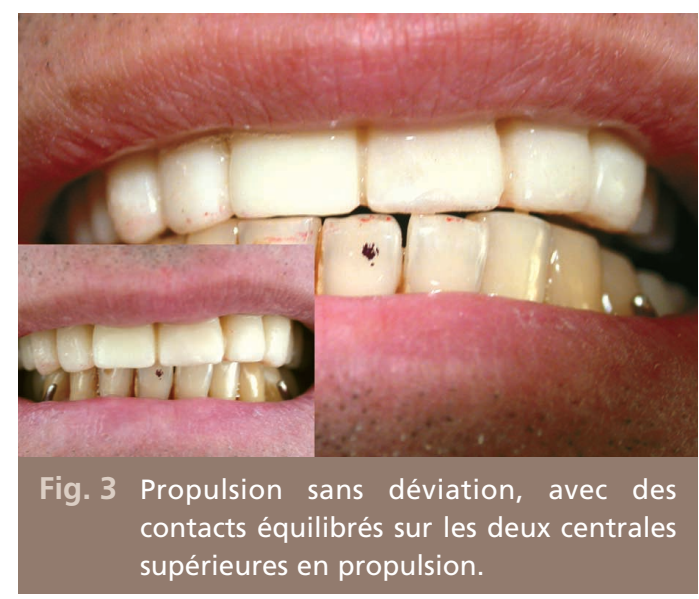

rapport à l'axe sagittal médian. Cet entonnoir assure la désocclusion postérieure par une prise en charge continue du déplacement, sur toutes les dents antérieures ou par relais, sans changement de sens[10], selon des contacts localisés aux crêtes marginales (économie de structure)[3, 11]. La position mandibulaire en bout à bout doit être équilibrée sur au moins deux incisives centrales sans induire de déviation mandibulaire (fig. 3)[12].

\section{$>$ En latéralité}

Qu'il s'agisse d'une fonction canine ou d'une fonction de groupe, la prise en charge du mouvement de latéralité doit être assurée sans interférences non travaillantes ${ }^{1}$. Les pentes de guidage doivent être symétriques par rapport au plan sagittal médian favorisant une mastication unilatérale alternée[12]. L'architecture occlusale doit assurer un déplacement condylien physiologique vers l'avant sans favoriser les rétro fonctions[2].

\section{> Liberté des mouvement mandibulaires}

Le concept d'une fonction antérieure efficace voir abrupte a évolué durant la dernière décennie, vers la description d'une fonction antérieure plus douce qui, tout en assurant une désocclusion postérieure, laisse une certaine liberté fonctionnelle antérieure (Slavicek)[4].

Le champ fonctionnel délimité par les dents antérieures détermine la valeur fonctionnelle du guide antérieur et dépend des valeurs de recouvrement, de surplomb de l'axe inter coronaire et de la morphologie palatine du bloc incisivo-canin maxillaire.

\footnotetext{
1 Une interférence non travaillante est caractérisée par la présence d'un contact occlusal non travaillant sur une pluricuspidée sans contact travaillant simultané.
} 


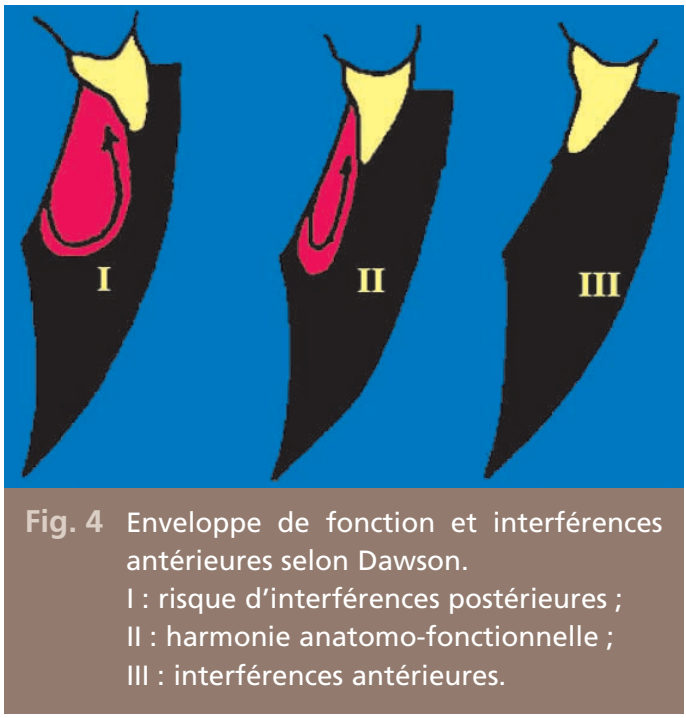

Il doit être assez fermé pour en assurer une désocclusion minimale, et assez ouvert pour une liberté fonctionnelle et un minimum de contraintes parodontales, sans quoi, un déplacement postérieur des cycles mandibulaires augmente le risque d'atteintes musculaires, articulaires et parodontales[13].

Le champ fonctionnel est borné en haut par les faces palatines des dents antérieures qui constituent la limite supérieure de l'enveloppe de mouvement. Cette enveloppe doit être en harmonie avec l'enveloppe de fonction correspondant à la superposition des cycles masticatoires du patient (fig. 4). Les deux enveloppes se rencontrent à la fin du cycle masticatoire alors que se réalise le mouvement de glissement mandibulaire vers I'OIM[13].

La validation d'un champ fonctionnel doit tenir compte de la notion de posture[14, 15] : régler les contacts occlusaux, patient en position allongée, est une erreur à éviter. Une fois redressé, la mandibule emprunte une position plus antérieure laissant apparaître des contacts antérieurs exagérés en statique et dynamique.

\section{Diagnostic et restauration du guide antérieur}

Dans l'état actuel de nos connaissances, il est impossible d'établir un lien de cause à effet entre une malocclusion et un dysfonctionnement voire une pathologie de I'ATM. La réhabilitation du guide antérieur pour des raisons prophylactiques ne peut donc être préconisée. Par contre, s'il existe d'autres raisons d'intervenir sur les dents antérieures, il faudra supprimer un facteur occlusal susceptible de favoriser un dysfonctionnement de l'appareil manducateur en rétablissant un guide antérieur fonctionnel[4, 15, 16]. Le diagnostic du guide antérieur prend alors toute son importance. Différentes situations cliniques peuvent être rencontrées :

- Guide antérieur fonctionnel : avec contacts antérieurs bien répartis, désocclusion postérieure et liberté des mouvements mandibulaires.

- Guide antérieur dysfonctionnel: le guide entrave la translation mandibulaire (classe II, division 2, où le champ de liberté fonctionnel est très réduit), ou est assuré par une seule dent. L'équilibration du guidage par des améloplasties soustractives ou additives le transforme en guide fonctionnel.

- Guide antérieur afonctionnel: présence d'interférences non travaillantes en propulsion et/ou latéralité (dents délabrées, béance antérieure, articulé inversé, surplomb excessif...). Il peut être restaurable ou non[17].

Face à ces situations cliniques, l'attitude thérapeutique est différente :

- intégration : face à un guide antérieur fonctionnel ou dysfonctionnel équilibré, si un traitement prothétique porte sur quelques 


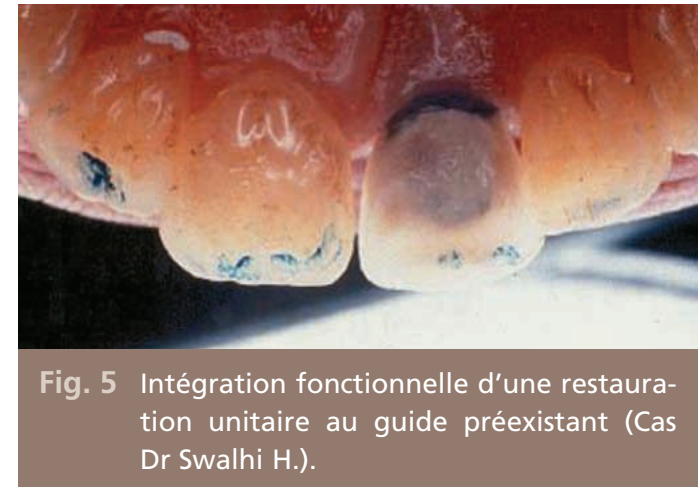

éléments antérieurs, leur morphologie palatine sera déterminée au laboratoire par rapport aux dents collatérales (fig. 5)[10] ;

- recopie : un guide antérieur fonctionnel doit impérativement être conservé et enregistré sur la table. Si un traitement prothétique de grande étendue affecte les dents antérieures, la réalisation d'une table incisive individualisée permet de retranscrire sur la prothèse définitive[10], la forme et l'orientation de la morphologie palatine des dents antéro-supérieures en toutes circonstances, pourvu qu'elle se situe sur la branche supérieure de l'articulateur[18] ;

- reconstruction : lorsque dents antérieures sont très délabrées, absentes ou si le guide antérieur existant ne permet pas d'effectuer les différentes fonctions, la recréation d'une anatomie palatine fonctionnelle, compatible avec la physiologie fonctionnelle et articulaire, se voit

\section{Cas clinique}

Un abord du cas, étape par étape, assure une approche logique et systématique, et se fait selon trois phases, évaluation, élaboration puis concrétisation.

\footnotetext{
$2 \mathrm{PI}$ : Pente incisive.

3 PC : Pente condylienne.
}

confrontée au problème très controversé d'interdépendance des déterminants antérieurs et postérieurs de l'occlusion[11, 19, 20).

Dans ce cas, l'utilisation de l'axiographie permet de déterminer la valeur de la pente incisive par rapport à la pente condylienne selon la formule $\left(\mathrm{PI}^{2}=\mathrm{PC}^{3}+10^{\circ}\right)$, la pente incisive adoptée est ensuite modulé en fonction de facteurs anatomiques (plan d'occlusion, pente cuspidienne, courbe de compensation)[4]. Cette méthode se trouve souvent confrontée à la méthode du tout en bouche préconisée par Dawson pour qui "On ne peut envisager d'établir et de régler les contours du guidage antérieur sur des bases exclusivement techniques»[10]. Cet auteur préconise en effet, de régler en bouche, les composants architecturaux du guide antérieur, tenant compte des différents impératifs esthétiques, phonétiques...

Entre partisans et opposants, et en l'absence de tout consensus sur les interrelations possibles entre déterminants antérieurs et postérieurs de l'occlusion, I'utilisation et la correction en bouche de provisoires de seconde intention, durant une période dite de temporisation, paraît la plus apte à vérifier l'intégration et la fonctionnalité de l'anatomie adoptée. Une fois validée, la recopie de la situation testée favorablement, permet de guider le prothésiste lors de la réalisation prothétique[11, 12].

\section{Phase d'évaluation}

Correspond à la collecte des informations. Elle permet d'établir un diagnostic et des objectifs de traitement.

Monsieur H. M. âgé de 48 ans en bonne santé générale, ancien fumeur, nous a été adressé 
après assainissement parodontal (fig. 6 a) pour une réhabilitation esthétique et fonctionnelle.

L'examen du patient révèle des douleurs musculaires aux temporaux, avec une faible exposition des dents antérieures à l'état de repos (fig. 6 b). L'hygiène bucco-dentaire du patient est satisfaisante. Les dents résiduelles (fig. 6 c) présentent une abrasion généralisée induisant

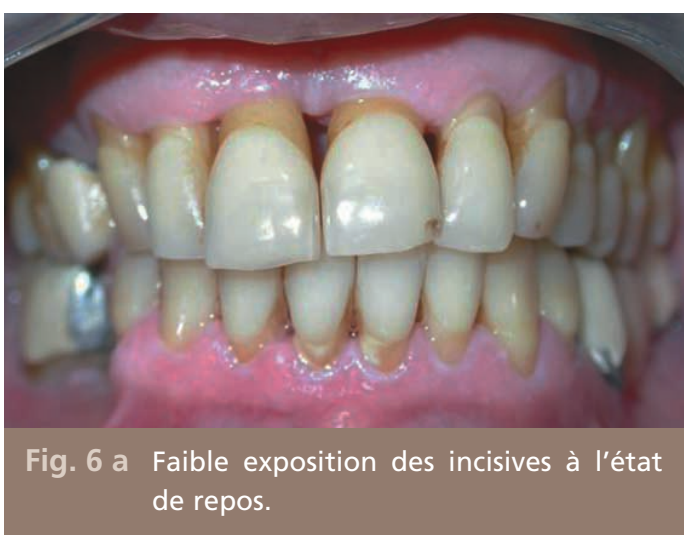

une OIM instable. Le guide antérieur est dysfonctionnel : le mouvement de propulsion est en effet pris en charge par la seule 11 qui de plus présente une mobilité degré 3 associée à un frémitus (fig. 7). L'examen parodontal montre une parodontite chronique généralisée de sévérité moyenne, des mobilités pathologiques sur 21 (degré 1), 22 (degré 1), 38 (degré 2), 48 (degré 2).

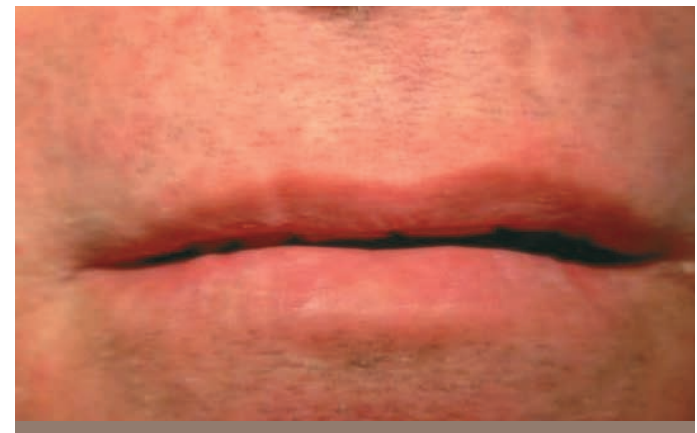

Fig. 6 b Vue frontale de la situation initiale.
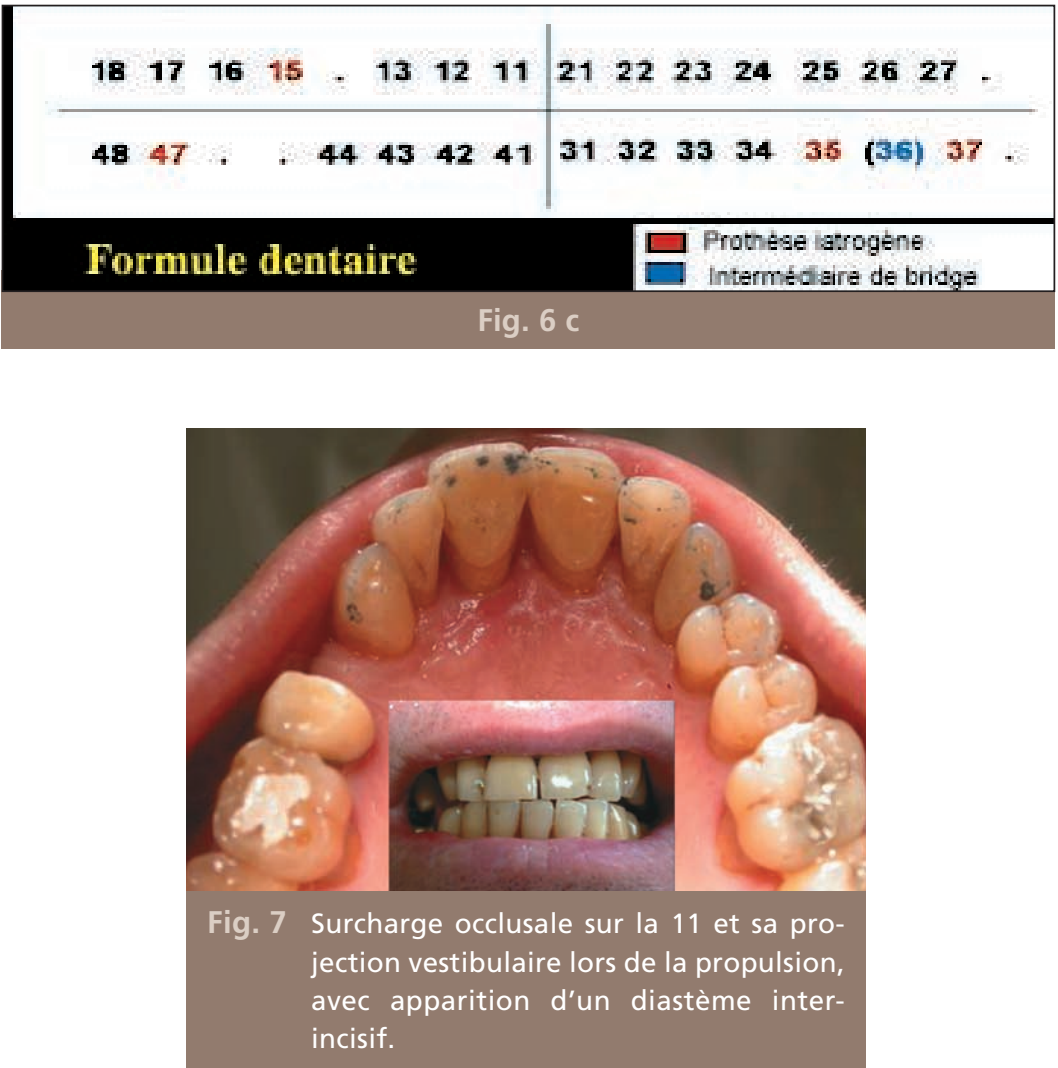


\section{> L'examen par radiographie rétro-alvéolaire révèle :}

(fig. 8)

- des traitements endodontiques défectueux sur $16,15,37,47$;

- une fracture de lentulo sur racine mésiale de 47 ;

- une réaction périapicale sur 16, 37, 48 ;

- une lyse osseuse horizontale généralisée, atteignant le tiers cervical sur le secteur postérieur maxillaire droit et la moitié des hauteurs radiculaires dans les autres secteurs ;
- une lyse osseuse angulaire terminale sur 11.

\section{$>$ Examen des moulages}

Les modèles sont montés sur articulateur à la bonne DVO et en relation centrée. L'évaluation de la situation du plan d'occlusion est facilité par la technique du drapeau décrite par Orthlieb[21] (drapeau positionné sur le même plan vertical que I'hémi-arcade à restaurer) et permet de matérialiser le degré d'égression sur 15, 16, 17 et 25,26 (fig. 9).
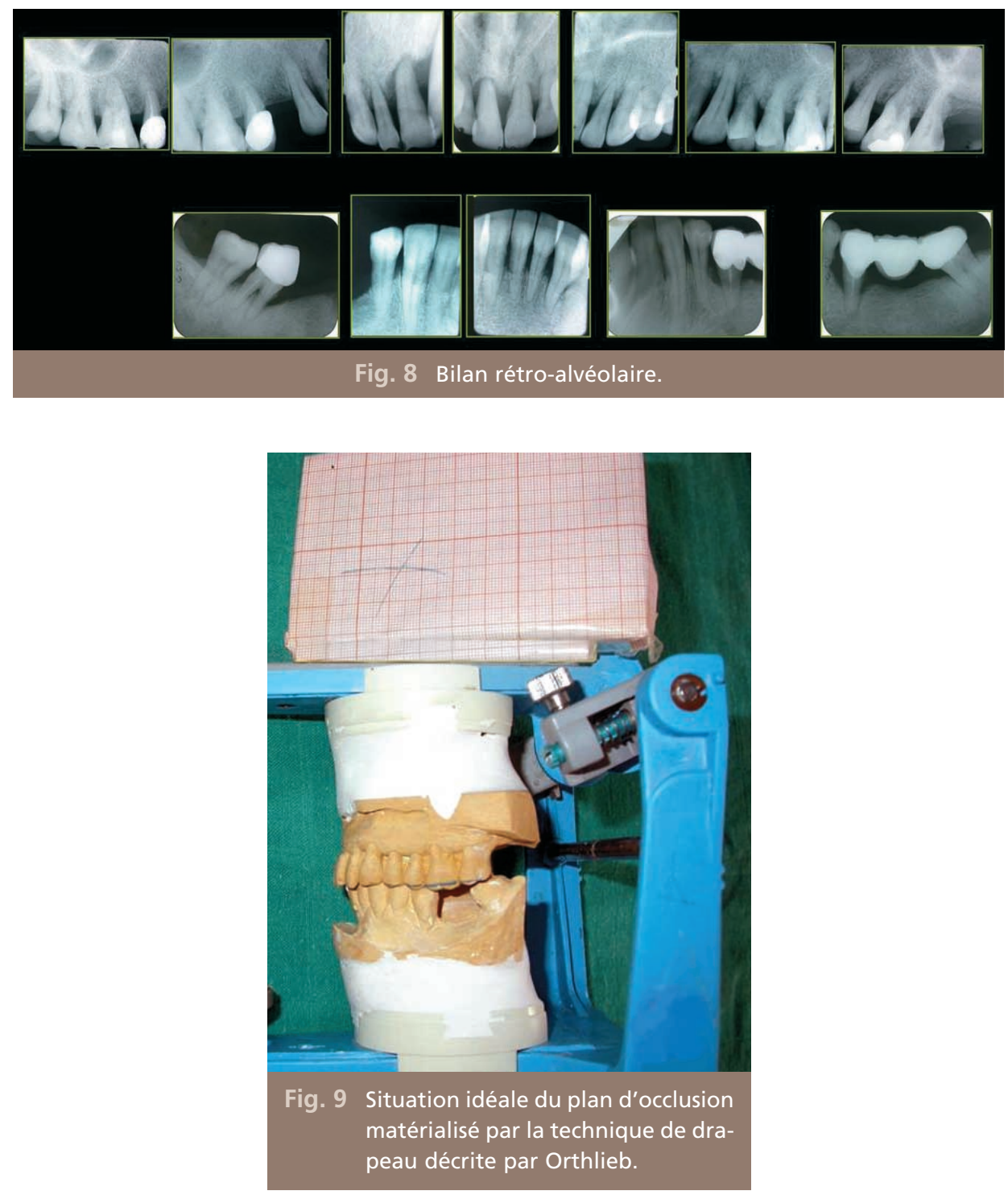


\section{> Objectifs de traitement}

- Rétablir un calage postérieur stable et centré à une dimension verticale d'occlusion physiologique.

- Rétablir un guidage antérieur fonctionnel permettant une liberté des mouvements mandibulaires, sans interférences antérieures ou postérieures.

- Maintenir l'état de santé parodontal.

Ces impératifs ne peuvent être respectés que par l'instauration d'une aire de liberté fonctionnelle conciliant fonction occlusale et résistance parodontale. Le recours à un certain nombre d'aménagements spécifiques au parodonte affaibli, améliore le pronostic de la restauration :

- contention par exploitation de plans de mobilité préférentielle différents et tenant compte du principe de la dent terminale (fig. 10 a). Ainamo et Alcoforado[9] affirment que c'est la solidarisation par des artifices rigides qui répartit les efforts, et non le choix de contacts occlusaux distribués au plus grand nombre de dents : exploiter des plans de mobilité préférentiel différents (fig. 10 b) permettrait une meilleure répartition des charges occlusales, les contraintes horizontales seraient ainsi minorées[22] ;

- instauration d'une morphologie palatine spécifique[7,9]: les forces transmises aux incisives supérieures, durant les mouvements mandibulaires, sont perpendiculaires à l'orientation de leurs faces palatines. Lorsque ces faces sont planes, la composante horizontale des forces est prédominante. Creuser les faces palatines des dents permet de réorienter les forces occlusales dans l'axe des dents à proximité des contacts en OIM (fig. 11) ; l'orientation des contraintes devenant horizontale à proximité du bord libre serait compensée par la qualité proprioceptive des dents antérieures, et leur grande sensibilité directionnelle. «Le seuil de détection de contact étant nettement plus bas pour des forces à direction horizontale que pour des forces dirigées le long du grand axe de la dent» (Orthlieb)[11]. La transmission de cette
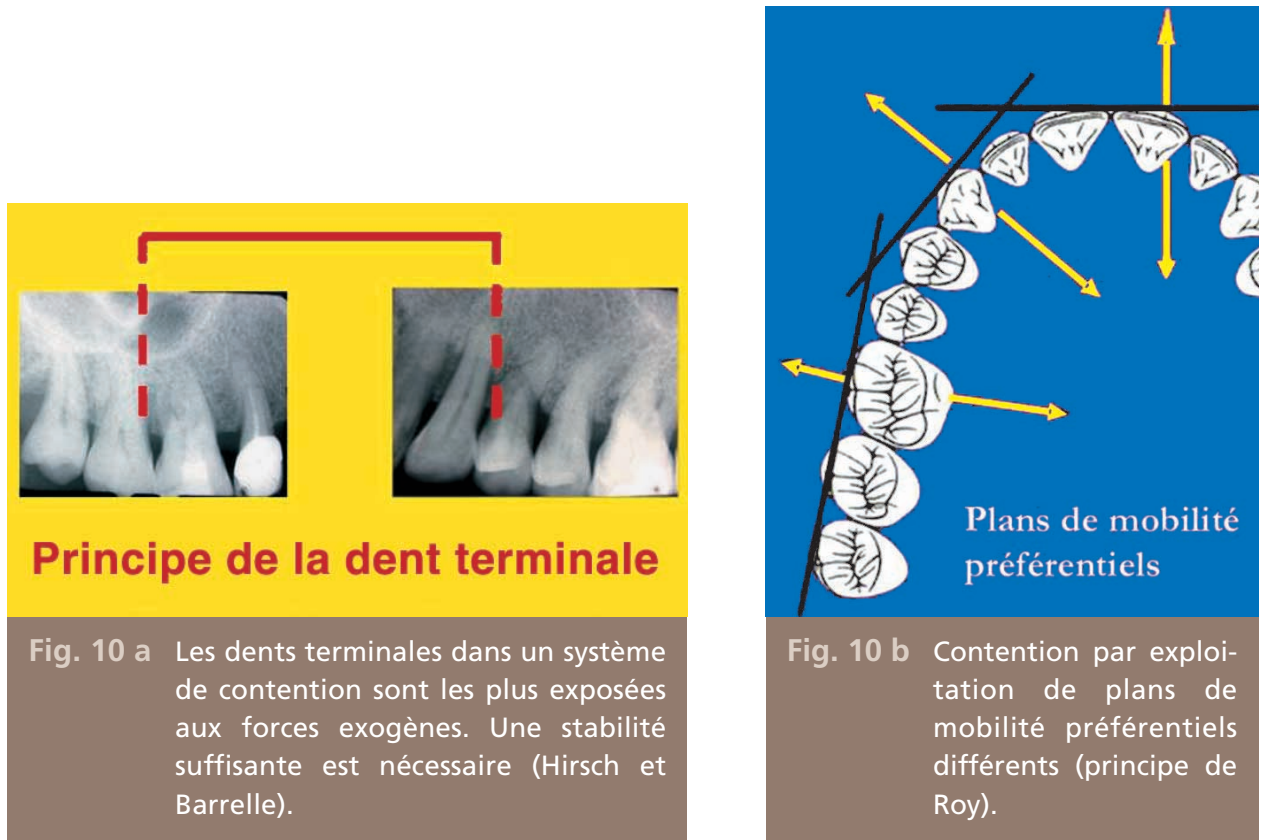

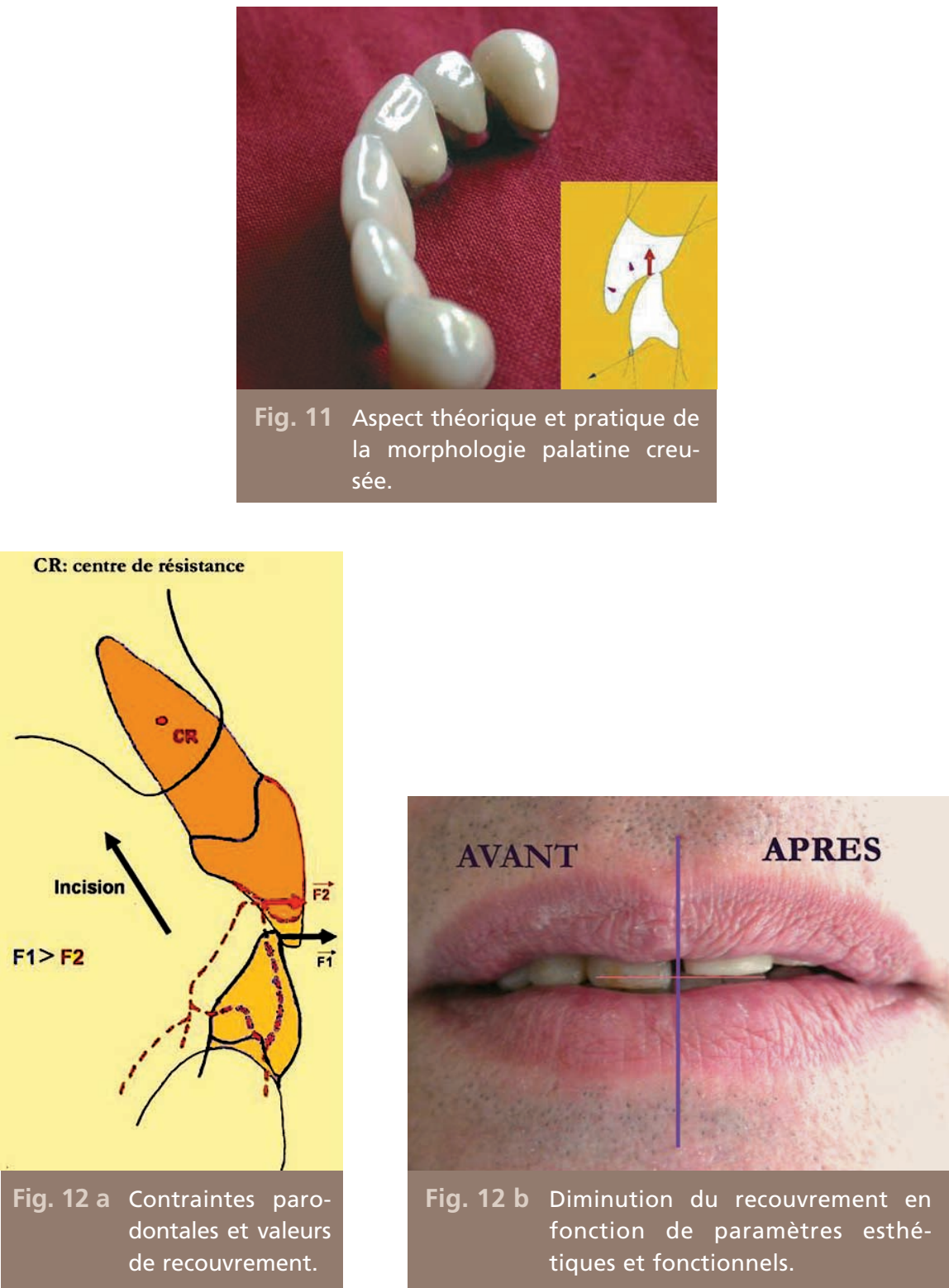

information tactile déclenche le plus souvent des réflexes d'évitement et une réduction significative de la capacité de contraction des muscles élévateurs ;

- diminution du recouvrement pour une réduction des contraintes en dynamique par raccourcissement du bras de levier exercé sur la dent (fig. 12 a et 12 b). Cette modification devra être en harmonie avec les déterminants postérieurs de l'occlusion[20] pré- servant le système de levier de troisième genre[2, 8], I'esthétique et la phonation «Fe, Ve». Cette réduction est contre indiquée dans notre cas pour des raisons esthétiques[2, 8, 9, 20].

\section{> Décision thérapeutique}

Un bridge céramo-métallique de contention de 16 à 23, associé à une prothèse composite inférieure. 
Phase d'élaboration

du projet prothétique

Elle correspond à la matérialisation en cire du projet prothétique.

"Ill y'a souvent controverse entre praticien et patient sur ce que doit être le résultat final. Lors du traitement de problèmes occlusaux, les phases prothétiques ne doivent pas être envisagées tant que le résultat final n'a pas été visualisé» Dawson[23].

- L'équilibration du guidage est réalisée en bouche après contention provisoire de la 11 . Les modèles d'études montés sur articulateur permettent alors la pré-programmation de la

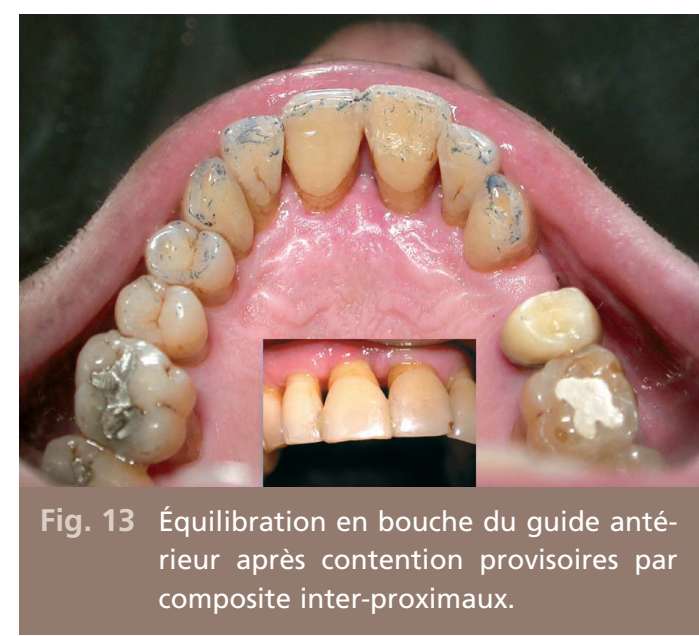

table incisive pour la reproduction de la forme et orientation des faces palatines sur les prothèses provisoires (fig. 13 et 14).

- L'empreinte composée globale de l'arcade inférieure préserve la précision et la pérennité des rapports occlusaux déterminés sur articulateur[24].

- Des cires de diagnostic (wax-up, et montage directeur) sont réalisées selon le plan d'occlusion idéal et la programmation de la table incisive préalablement déterminés. Ils assurent une désocclusion postérieure en propulsion, et désocclusion non travaillante en latéralité selon des contacts antéro-latéraux (fig. $15 \mathrm{a}, 15$ b et $15 \mathrm{c}$ ).

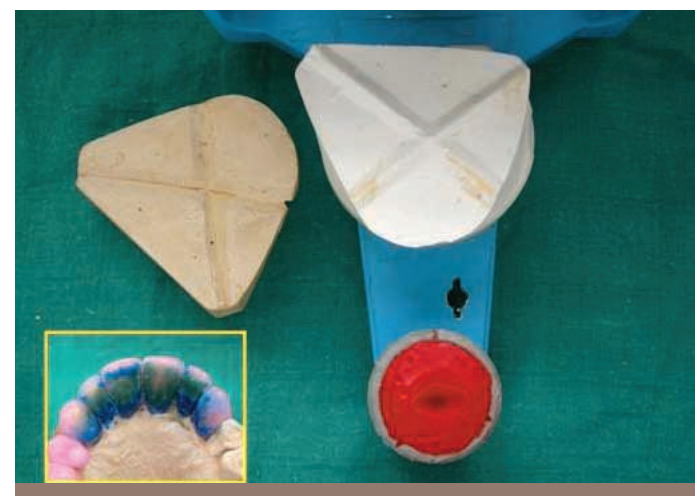

Fig. 14 La pré programmation de la table incisive retranscrit sur les wax-up les paramètres de guidage enregistrés en bouche.

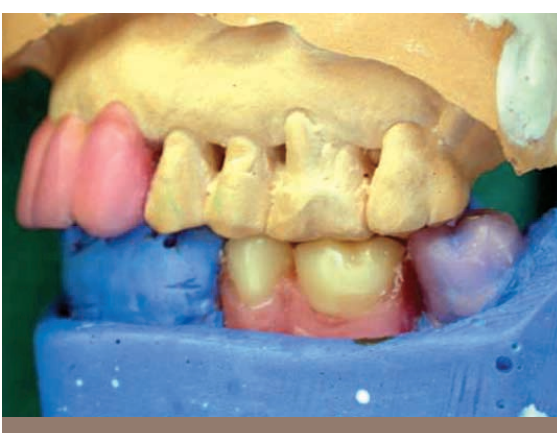

Fig. 15 a Situation du plan d'occlusion secteurs 2/3: le compromis préserve l'intégrité du complexe pulpo-dentinaire.
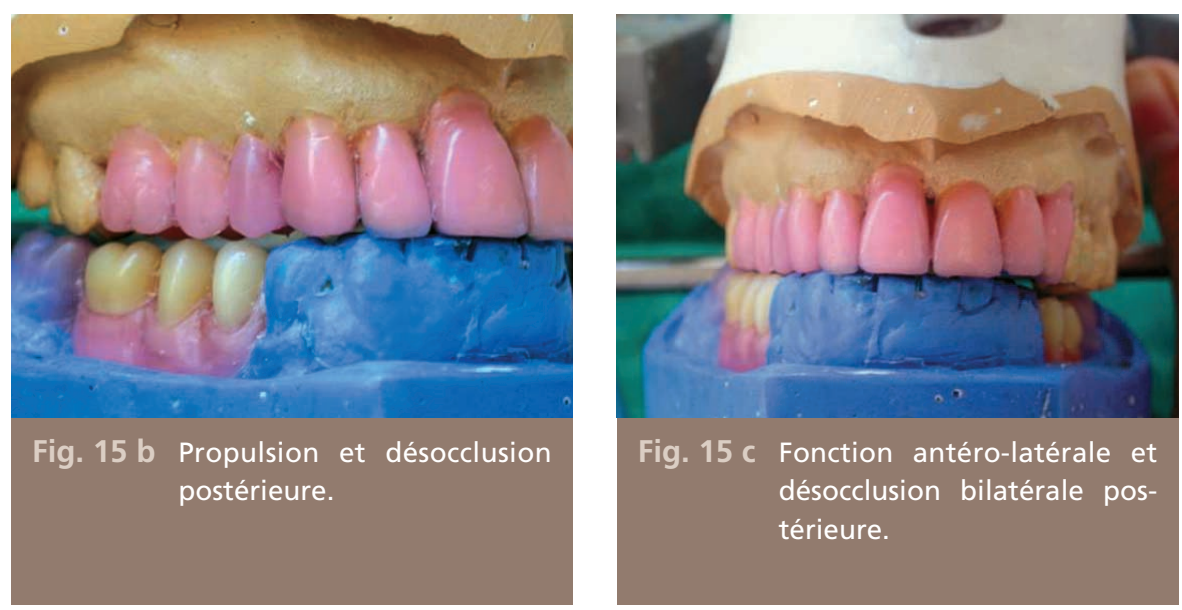

Fig. 15 c Fonction antéro-latérale et désocclusion bilatérale postérieure. 


\section{Phase de concrétisation}

Elle correspond au transfert en bouche des modifications obtenues sur modèles d'étude (fig. 16).

- Les provisoires issues des modèles de diagnostic sont mises en place dans le cadre d'une thérapeutique étiologique comprenant, détartrage, dépose des prothèses iatrogènes sur $15,35,36,37$, et 48 , soins des caries, extraction de 11, 35, et 47 jugées irrécupérables, traitements endodontiques pré prothétiques sur 12,22 et 48 , et reprises de traitements endodontiques sur $16,15,37$, puis mise en place d'un bridge provisoire de contention de 16 à 23 et d'une PAP résine inférieure pour une stabilité occlusale optimale.

- Une phase de «rodage clinique»[12] permet par équilibrations successives des facettes d'abrasion fonctionnelles palatines, d'obtenir des trajectoires de guidage en harmonie avec l'enveloppe de fonction (fig. 17 a). Le guidage assure une désocclusion postérieure minimale (fig. 17 b) selon des trajectoires harmonieuses contrôlées en propulsion et en rétropulsion (fig. 17 c) : I'utilisation du seul mouvement de propulsion pour vérifier les guidages fonctionnels du secteur antérieur, risque de laisser
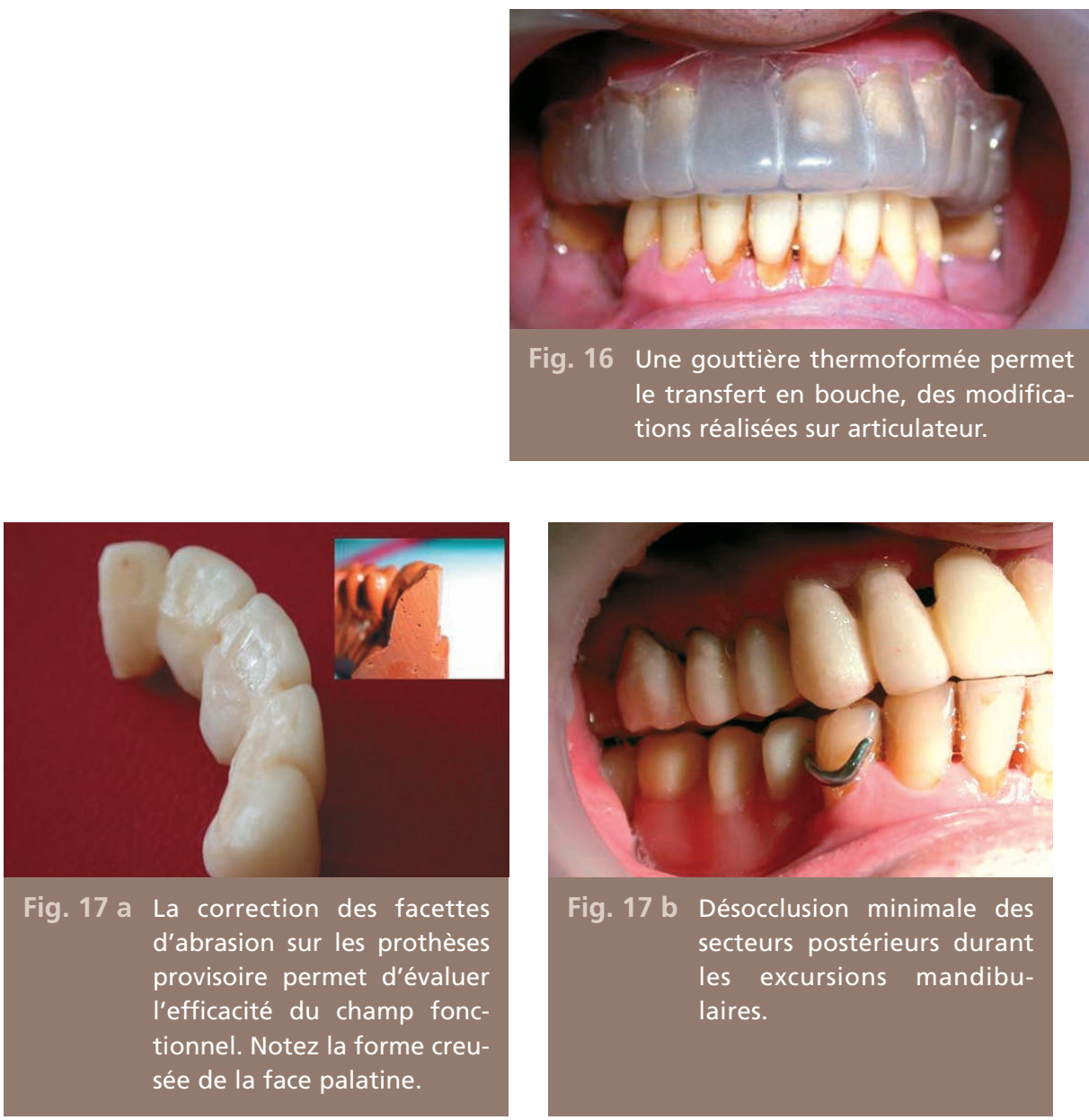

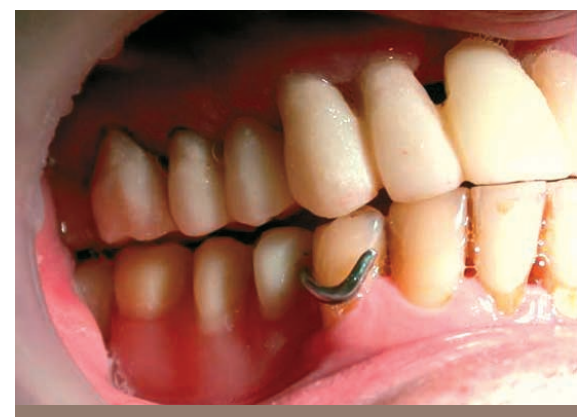

Fig. 17 b Désocclusion minimale des secteurs postérieurs durant les excursions mandibulaires.

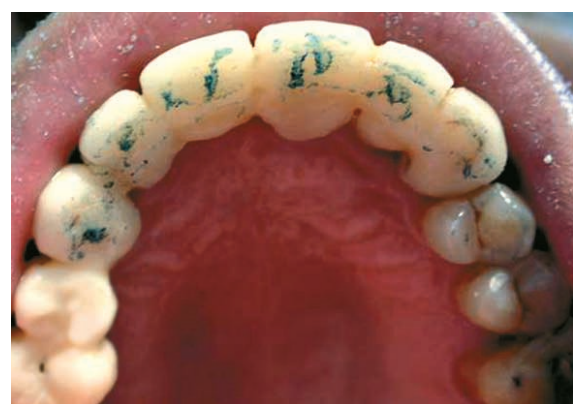

Fig. 17 c Trajectoire de guidage en propulsion, rétropulsion. 
des surguidages lors de l'incision, non apparents à la propulsion[3, 19, 26].

- Une période de temporisation permet de valider la proposition thérapeutique à la fois d'un point de vue esthétique et fonctionnel, d'évaluer la stabilité des lésions parodontales, et d'obtenir le consentement éclairé du patient.

Cette période de temporisation associée à des prothèses provisoires de grande qualité représente la clé de la réussite prothétique[7, 15].

«La prothèse provisoire doit être affinée jusqu'à un degré tel que la seule différence des matériaux utilisés la distingue de la prothèse définitive.»

Sabek[7]

"L'établissement d'une occlusion physiologique chez des patients candidats aux restaurations complexes, est généralement envisagée lors de la réalisation des prothèses provisoires, c'est-à-dire au stade de temporisation.» Keough[15]
Cette période de temporisation précède la réalisation des reconstructions prothétiques d'usage :

- l'arcade inférieure est restaurée en premier lieu par une prothèse composite associant des chapes de morphologie adaptée (fig. 18 a) porteuses d'attachements boutonpression à un châssis métallique (fig. 18 b). Cette prothèse assure un calage postérieur précis et stable dans les mêmes rapports intermaxillaire testés par les prothèses provisoires (fig. $18 \mathrm{c}$ ). Les chapes rétablissent un rapport couronne clinique/racine clinique favorable sur les piliers postérieurs améliorant leur pronostic parodontal ; elles évitent les inconvénients de l'édentement en extension, empêchant le mouvement de rotation distal terminal pour un équilibre occlusal optimal, et assurent un meilleur équilibre prothétique ;

- la restauration de l'arcade maxillaire passe par le transfert précis au laboratoire, des informations obtenues sur prothèses provisoires :

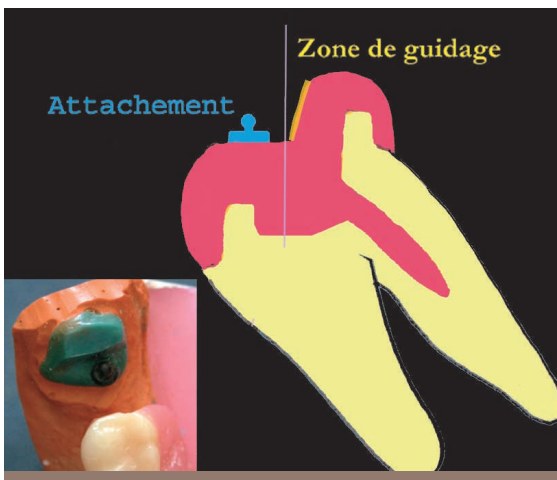

Fig. 18 a Chape à morphologie adaptée comprenant une plate forme horizontale de sustentation, un attachement type bouton pression et une zone de inclinée de guidage et de stabilisation.

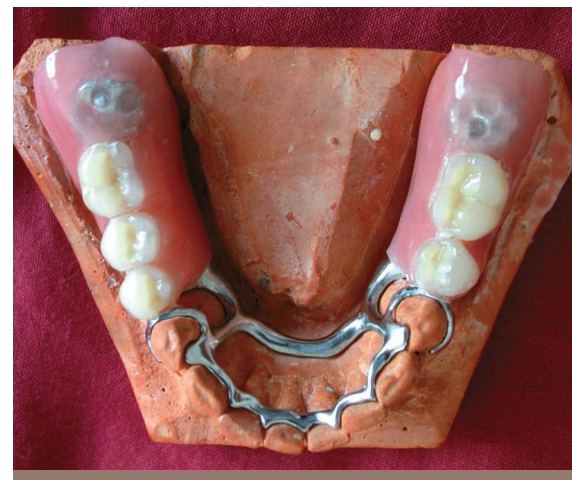

Fig. 18 b La conception semi-rigide du châssis au niveau des piliers antérieurs anticipe la perte éventuelle des piliers terminaux et la transformation en édentement terminal.

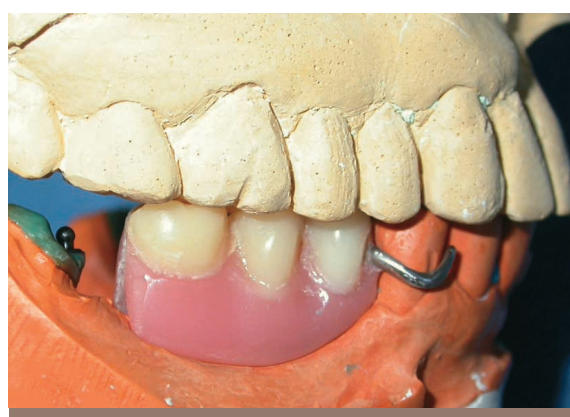

Fig. $18 \mathrm{c}$ La prothèse provisoire du patient est entraînée dans l'empreinte secondaire des chapes et assure le transfert précis du RIM. 
- après fractionnement du bridge provisoire, des mordus alternés permettent le transfert des modèles de travail dans le rapport intermaxillaire validé lors de la période de temporisation (fig. 19 a) ;

- le montage sur articulateur des modèles issus de l'empreinte des prothèses provisoires en place, permet la programmation de la table incisive selon les données individuelles du patient, conservant ainsi les mêmes formes et orientations palatines que celles des provisoires[7] (fig. 19 b) ;

- des clés vestibulaires et palatines, en silicone, permettent de guider le prothésiste

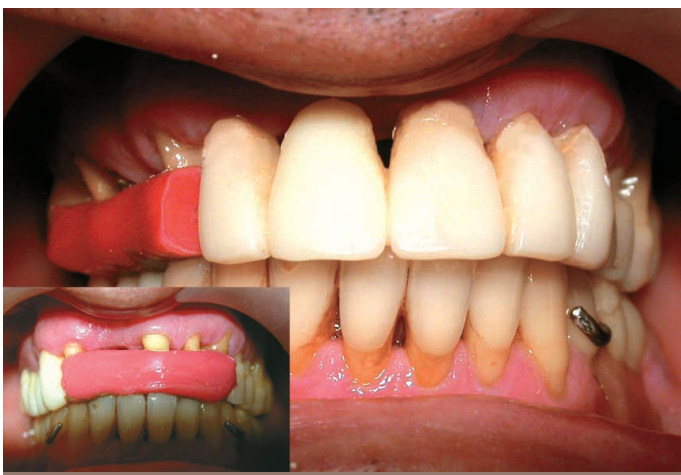

Fig. 19 a Mordus alternés en cire MOYKO rebasée par du ciment de scellement provisoire.

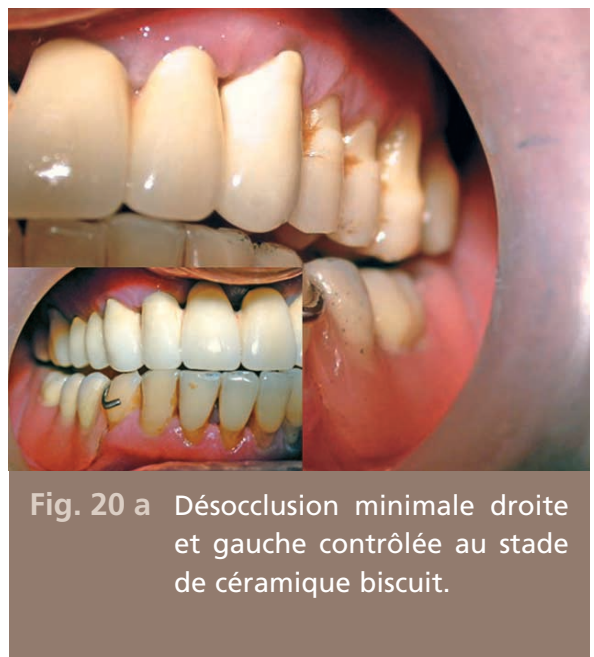

lors du choix de la forme, des dimensions dentaires, de la situation du point interincisif... (fig. 19 b) ;

- les contacts occlusaux et la désocclusion minimale sont contrôlés en bouche (fig. 20 a) ; la situation supra gingivale des lignes de finitions n'altère pas le rendu esthétique du fait de la situation basse de la ligne du sourire (fig. 20 b) ;

- le scellement définitif est assuré par un oxyphosphate de zinc, sous occlusion ;

- un programme de maintenance est établi en collaboration avec le patient.
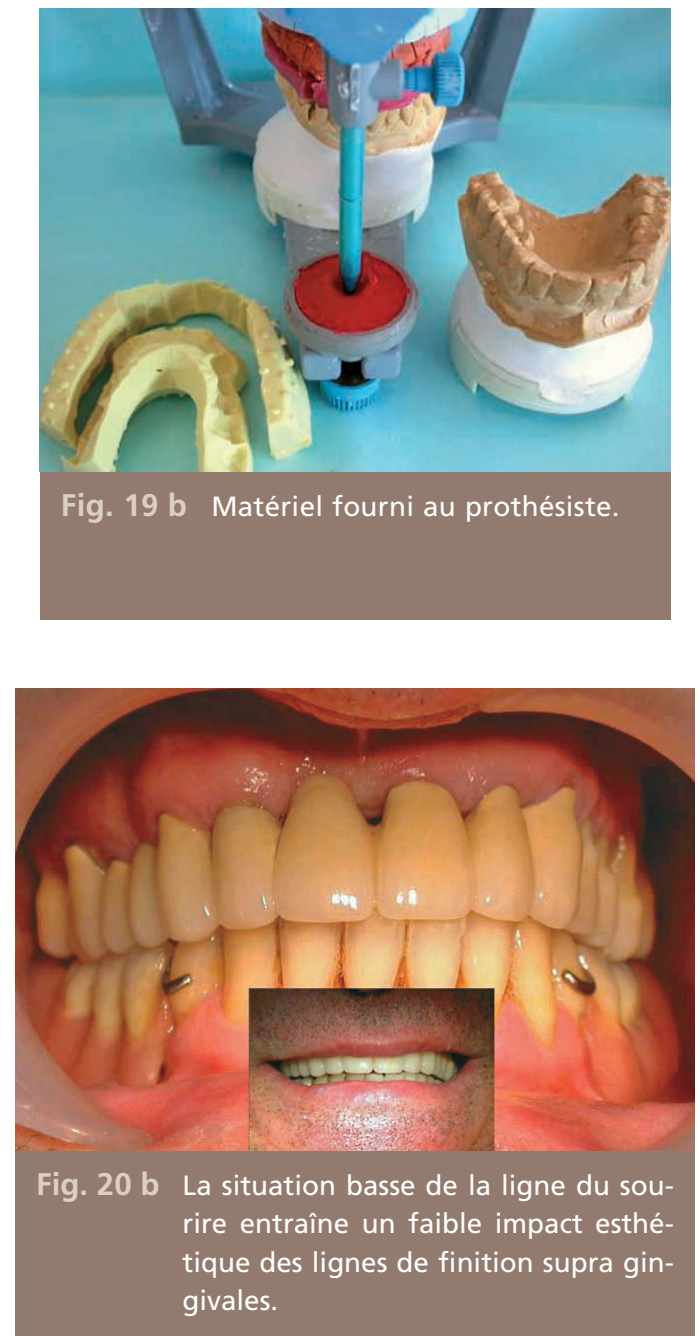


\section{Discussion}

La restauration d'un guide antérieur fonctionnel et protecteur est une condition sine qua non à la réussite et à la pérennité de tout traitement prothétique. Cet objectif ne peut être atteint que par l'examen et le diagnostic précis des rapports d'occlusion entretenus par les dents antérieures, associés à une démarche thérapeutique raisonnée et validée par une phase de test. La programmation de la table incisive selon des données individuelles assure le transfert précis des paramètres de guidage au laboratoire pour une intégration prothétique optimale.

Cette approche fonctionnelle est pourtant confrontée à la divergence des points de vue existants entre praticien et patient sur ce que doit être le résultat final[8]. Leur hiérarchisation des priorités est en effet différente: un praticien occluso-conscient privilégie par ordre d'importance décroissant la fonction, la résistance prothétique et l'esthétique; le patient, lui, adopte souvent l'ordre inverse[12]. II est, en effet, plus simple pour un patient d'intégrer une prothèse esthétiquement valable et fonctionnellement médiocre plutôt que le contraire, le compromis entre esthétique et fonction doit être recherché.

\section{Bibliographie}

1. Borel JC, Schittly J, Exbrayat J. Manuel de prothèse partielle amovible. 2 ed. Paris : Masson, 1994.

2. Hue $\mathrm{O}$.

L'occlusion :

aspects anatomiques, aspects neurophysiologiques. Cah Prothèse 1997; 100:65-75.

3. Laplanche $\mathrm{O}$, Pedeutour $\mathrm{P}$, Laurent M,.Mahler $\mathrm{P}$, Orthlieb JD.

Le guide antérieur et ses anomalies. Incidence sur la cinématique condylienne.

Cah Prothèse 2002;117:43-55.

4. Laurent. M, Orthlieb JD. Approche occlusale d'une restauration des incisives maxillaires. Cah Prothèse 1997;99:11-19.

5. Valentin CM, Yakou O. Reconstruction du guide antérieur par des artifices de prothèse conjointe. Réalités cliniques 1993;2: 163-176.

6. Lubespere $A$, Jacquet $E$, Cretot M.

Le guide antérieur en prothèse fixée. Quest. Odonto-stomat 1986;11:515-527.

7. Sabek M, Trevelo A. Restauration du guide antérieur. Impératifs et moyens techniques.

Rev Odonto-stomat 1994;4:291-309.

8. Dawson PE. Les problèmes de l'occlusion clinique. Paris, Edition Cdp, 1992.

9. Ainamo J, Alcoforado $G$, Borgetti A, Guyot JF. Reconstruction du guide antérieur sur parodonte réduit.
Actualités Odonto-stomat 1996; 194:275-287.

10. Renault P, Pierrisnard L. Occlusion et prothèse fixée, démarche décisionnelle et principes du plan de traitement. Cah Prothèse 2000;112:63-82.

11. Orthlieb JD, El Zoghby A, Kordi M, Perez C. La fonction de guidage : un modèle biomécanique pour un concept thérapeutique. Cah Prothèse 2004;128:43-53.

12. Orthlieb JD, Bezzina $S$, El Zoghby A, Giraudeau A. Reconstruction prothétique du guide antérieur. Cah Prothèse 2004;128:55-63.

13. Le Gall MG, Lauret JF. Réalité de la mastication. Cah Prothèse 1998;103:13-20.

14. Keough B.

Occlusion

et prothèse parodontale. 
Revue internationale de parodontie et dentisterie restauratrice 1992;5:359-372.

15. Assaf A, Joubert E.

Le guide antérieur. Esthétique et fonction. Inf Dent 1994;24:2081-2093.

16. Dubreuil J, Trévelo A. Articulateurs et prothèse adjointe totale. Actualités Odonto-stomat 1992;177:113-134.

17. Le Deschault $P$. Anomalies du guide antérieur : conséquences et réhabilitation en prothèse fixée. Th D : Chir Dent, Bordeaux 2002, N 27.

18. Orthlieb JD, Laborde G, Bezzina S, Gros P. Rôles de I'articulateur dans la transmission des données du cabinet au laboratoire. Réalités Cliniques 2002;13:109-123.
19. Michielin $M$, Damiani MG, Orthlieb JD, Simon J. Analyse statistique des interrelations fonctionnelles entre le guide antérieur et déterminant postérieur. Cah Prothèse 1990;70:53-66.

20. Feuillerat $C$, Simon J, Orthlieb JD. Morphologie fonctionnelle des incisives maxillaires, étude statistique préalable. Cah Prothèse 1988; 63:83-93.

21. Orthlieb JD.

La courbe de Spee : un impératif physiologique et prothétique.

Cah Prothèse 1983;44:89-116.

22. Ainamo J, Alcoforado G, Borgetti A. Coll. Maladie parodontale, mobilité, contention, et prothèse. Actualités Odonto-stomat 1996;194:245-258.

23. Dawson PK. Solving occlusal problems through programmed treatment planing. In: Evaluation, diagnosis and treatment of occlusal problems. St louis: Mosby 1970:387.

24. Schittly J, Borel JC, Exbrayat J.

L'occlusion en prothèse partielle amovible. Réalités cliniques 1995;6:447-465.

25. Laplanche O. J.6 Sarlin J, Torque G, Zerbib C. Contrôle clinique de l'intégration occlusale d'une prothèse fixée. Cah Prothèse 2004;128:31-42.

26. Le Gall MG, Lauret JF. Occlusion et fonction : une approche clinique rationnelle.

Paris : éditions CDP, 2002.

27. Lasserre JF, Sbus M, Leriche MA. Les moyens de communication entre praticien, patient et prothésiste.

Cah Prothèse1999;108:75-82.

\section{SUMMARY}

\section{Anterior guide, physiological requirements and prosthetic restauration}

Omar IRAQUI,

Salwa BERRADA,

Nadia MERZOUK

Ahmed ABDEDINE

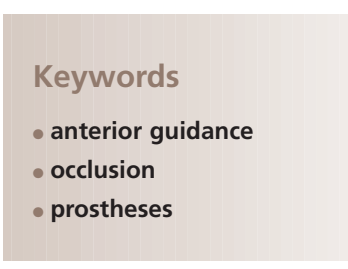

The masticatory system balance is crucial for any occlusion-conscious physician. It requires the restauration of a functional and protective anterior guidance. This goal cannot be reached without the examination and diagnosis of previous occlusal relations compared to a reference academic model.

The therapeutical pattern will be modulated based on gathered information, and requires the integration, or the regeneration of the guidance. Whatever the sequence of the treatment is, the validation of the guidance parameters through temporary prostheses, during the temporizing and transcripting phase of the data on the permanent prosthesis, is the only way to assure a prosthetic integration. This work outlines the benefits of a functional anterior guidance and the different ways to realize it based on a prosthetic restauration through a fixed prostheses. 